\title{
THE RATE OF RECOMBINATION OF THE IONS IN AIR.
}

\author{
By L. L. HENDREN.
}

IF a gas is ionized by any ionizing agent such as Röntgen or radium rays the positive and negative ions will immediately begin to recombine due to their motion and mutual attractions. A simple law, first suggested by J. J. Thomson and Rutherford ${ }^{1}$ and obtained by analogy from the law of mass action in physical chemistry, has been found to cover this phenomenon. It states that the rate of disappearance of either charge is proportional to the positive and negative electrical densities, $p$ and $n$. Thus

$$
\frac{d p}{d t}=\frac{d n}{d t}=-\alpha \cdot p
$$

where the proportionality factor $\alpha$ is called the coefficient of recombination.

If $p$ and $n$ represent the number of ions per c.c. instead of the charges on these ions the coefficient will be the $\alpha$ above times $e$, the charge on one ion.

Rutherford, ${ }^{2}$ McClung ${ }^{3}$ and Langevin ${ }^{4}$ have investigated this law with the conclusion that it is a true one, while Townsend, ${ }^{5}$ McClung and Langevin have determined the value of the coefficient $\alpha$ for several gases, agreeing with each other as to its value well within the experimental error at atmospheric pressure. For the variation of $\alpha$ with the pressure however, which has been taken up for air by McClung and Langevin, the agreement has not been so good; McClung coming to the conclusion that the coefficient of recombi-

\footnotetext{
${ }^{1}$ Phil. Mag., V., 2, p. 92, $89^{6}$.

2 Phil. Mag., Nov., 1897, p. 44 ; Jan., 1899.

${ }^{3}$ Phil. Mag., March, 1902.

4 Thèse prèsentée à la Faculté des Sciences.

5 Phil. Trans. Roy. Soc., p. 157, 199.
} 
nation is independent of the pressure, while Langevin obtained a rapid decrease.

The purpose of the present investigation was to obtain additional data as to the variation of the coefficient of recombination with the pressure, also to obtain values below the limits of pressure previously used.

The results of the work given in a later paragraph show that $\alpha$ decreases with the pressure, though not so rapidly as Langevin's results would indicate. Determinations were made down to IO $\mathrm{mm}$. pressure as compared with $\mathrm{I} 00 \mathrm{~mm}$. and $\mathrm{I} 50 \mathrm{~mm}$., the limits reached by McClung and Langerin respectively. Values of the coefficient of diffusion of the ions were also obtained at 20 and IO $\mathrm{mm}$. pressure.

\section{Method of McClung.}

Calling $q$ the total charge on the ions, positive or negative, produced per second by the ionizing agent in each c.c. of the gas, and assuming that there is an equal number of positive and negative ions in each c.c.

$$
\frac{d n}{d t}=q-\alpha n^{2}
$$

Thus after the ionization begins the number of ions in the gas will constantly increase until a state of equilibrium is reached between the rate of recombination $\alpha n^{2}$ and the rate of production $q$, i. e., until $d n / d t=0$, when we have the relation

$$
\alpha=\frac{q}{n^{2}},
$$

or if $Q$ and $N$ represent the volume integrals of $q$ and $n$ in a certain volume $V$

$$
\alpha=\frac{Q}{N^{2}} K,
$$

where $K$ is a constant depending upon the dimensions of the vessel used as an ionization chamber, and determined by the distribution of the ionization. If the ionization is uniform $K=V$. Using this formula McClung made a direct determination of $\alpha$. His ionizing agent was the Röntgen rays which ionized the gas contained in a 
[Vol. XXI.

cylindrical vessel $20 \mathrm{~cm}$. long and separated into ten compartments by thin aluminum partitions. The alternate partitions were connected to each other and acted as electrodes. The $Q$ above, which is the saturation current, was determined in the usual way by noting, by means of the movement of an electrometer needle, the rate of charge of a known capacity connected in parallel with the electrometer and one set of electrodes, against which the ions were being driven by a saturation potential.

The instantaneous value of the free charge $N$ was measured by allowing the Rontgen rays to ionize the gas until a steady state was reached, then stopping the ionization by breaking the primary circuit of the induction coil, and simultaneously with the stopping of the rays, throwing on a saturation potential across the electrodes, thus driving all the ions present in the gas against the electrodes. The charge $N$ on these ions was obtained by noting on an electrometer the potential to which a known capacity was raised.

\section{Method of Langevin.}

Langevin's experimental determination of $\alpha$ is based on a very important theory in which he proves that practically all of the recombination of the ions can be accounted for by the consideration of their mutual attractions. On this basis he obtains the formula

$$
\alpha=4 \pi\left(k_{1}+k_{2}\right)=
$$

in which $\left(k_{1}+k_{2}\right)$ is the sum of the specific velocities of the ions and $\varepsilon$ is the fraction of the whole number of collisions which result in recombination. The $k_{1}+k_{2}$ was measured directly while $\varepsilon$ was obtained from experiments based on a further consideration of the theory of ionization. The velocities of the ions increase with decreasing pressure and the $\varepsilon$, due to the large kinetic energy of the ions for long free paths, decreases, thus theoretically it cannot be predicted whether $a$ should decrease with decreasing pressure or not. Langevin as stated before found a rapid decrease.

\section{Method and Description of the Apparatus.}

The method adopted in the present case is similar to that of McClung's in that it depends upon the formula 


$$
\alpha=\frac{Q}{N^{2}} K
$$

but differs from his in that radium was used as the ionizing agent and the $N$ was determined in a new way. For the determination of $N$, the charge on the ions present in the gas at any time after a steady state has been reached, it was not attempted to stop the ionization, but the electromotive force was allowed to act across the electrodes for varying intervals of time and the charges thus obtained were corrected for the rate of production during that time.

By the use of radium a great ionization compared with that possible from Röntgen rays can be obtained. Also the very serious difficulty of the inconstancy of the radiation, encountered by other observers in the use of Röntgen rays, is avoided, as the intensity of the radium radiations are very constant after the radium has reached a steady state. The chief objection to its use is the error brought in by the uneven distribution of the ionization due to the absorption of the rays. This will be discussed later.

The diagrammatic arrangement of the apparatus is shown in Fig. I.

$A$ and $B$ are two aluminum plates, respectively 26 and $30 \mathrm{~cm}$. in diameter, supported and insulated by hard rubber and mounted inside a large glass bell-jar. Both $A$ and $B$ were coated as uniformly and equally as possible with a solution of very active radium chloride, about one milligram of the salt being in the solution. In a majority of the experiments $A$ and $B$ were $6 \mathrm{~cm}$. apart. An aluminum ring $G, \mathrm{I} .5 \mathrm{~cm}$. wide, was placed around $A$ with $.5 \mathrm{~cm}$. space between. This ring was also coated with radium and acted as a guard ring, tending to make the ionization of the volume of gas under the plate $A$ more uniform with respect to the plane of the plates. $G$ was kept grounded so that only the ions directly under $A$ would be driven into $A$ by a potential on $B$. Those ions contained in the volume extending out half way between $A$ and $G$ were assumed to be caught by $A$, thus making the effective area 572 sq. $\mathrm{cm}$. The upper plate $A$ was connected through the key $K_{2}$ to the variable known capacity $C$ and this was connected through the key $K_{1}$ in parallel with the electrometer, which was of the 
[VoL. XXI.

Dolazalek type. For the purposes of the experiment it was necessary to be able to apply an electromotive force to the electrode $B$ for varying intervals of time. To accomplish this the plate $B$ was connected in series with a very high resistance to one terminal of a variable high potential source, the other end of which was grounded. A second circuit was run from $B$ to the earth through the breakcircuit key $T_{1}$ and the make circuit key $T_{2}$. Both $T_{1}$ and $T_{2}$ were

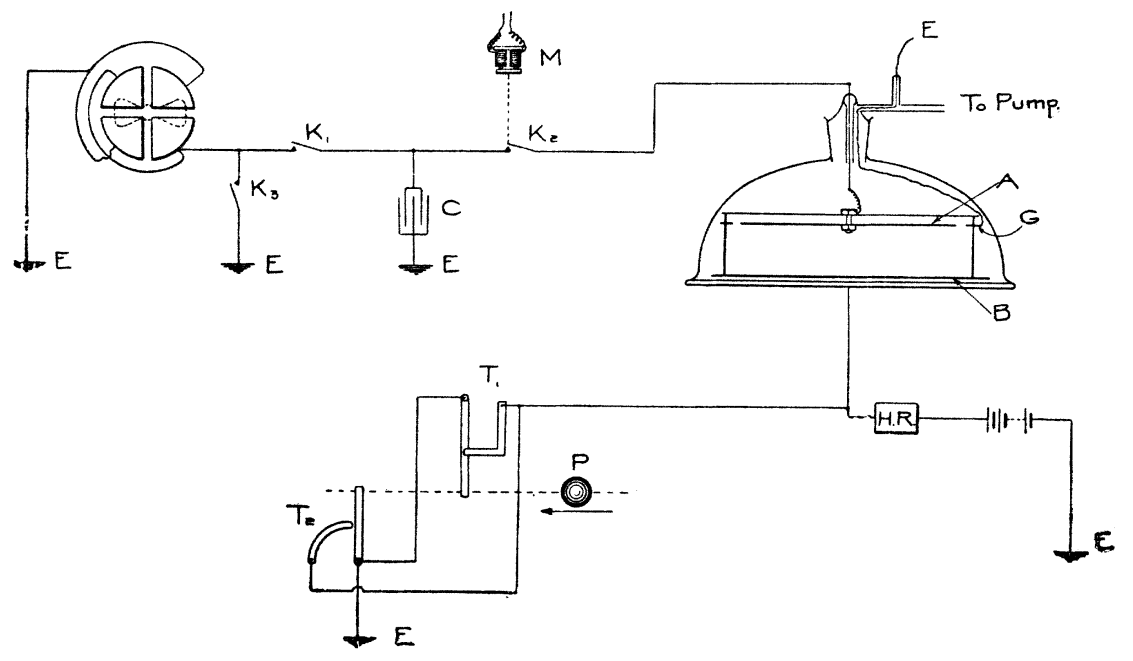

Fig. 1.

placed on adjustable mounts in the path of a pendulum swinging in the path indicated by the arrow. By this arrangement when either $T_{1}$ or $T_{2}$ was closed $B$ was at zero potential and at the potential of the battery for $T_{1}$ and $T_{2}$ both open. The pendulum arrangement was calibrated so that the electromotive force could be thrown across $B-A$ for intervals of time varying from . I to .4 second.

\section{Method of Observation.}

The saturation current $Q$ was obtained in the usual way by noting on the electrometer the rate of increase of the potential of the known capacity $C$ with the time. A correction was applied to allow for the leak from the plate $A$ to the guard ring. This correction was not large as $A$ was never charged to more than one half a volt. 
Care was always taken to have a sufficient potential across $B-A$ to drive out the ions as fast as they were produced, thus getting a true saturation current. Due to the large ionization at high pressures, the saturation potential was very high, 6,000 volts being necessary at atmospheric pressure for the plates $6 \mathrm{~cm}$. apart. The method of experimenting for the determination of $N$, the amount of free electricity on the ions after the gas has reached a steady state was as follows: First, the capacity $C$ was placed at a suitable value, the three keys, $K_{3}, K_{2}$ and $K_{1}$ were closed, as was also the break contact key $T_{1}$, thus grounding the system. $T_{2}$ was left open and a distance corresponding to . I sec. left between $T_{1}$ and $T_{2}$. The pendulum was now swung and at the same time the key $T_{1}$ was automatically opened, insulating the capacity and electrode $A$. When the pendulum opened $T_{1}$ the ground was removed and the potential of the battery was across $B-A$ for . I sec., until $T_{2}$ was closed. Thus a certain charge was thrown into the capacity, consisting of the quantity of free charge $N_{1}$ on the ions in the gas plus the amount on the ions produced by the radium radiation during. I sec. Simultaneously with the closing of the key $T_{2}$, another circuit, not shown in the figure, was closed, thus actuating the electromagnet $M$ which opened the key $K_{2}$ and so insulated the capacity $C$ containing the charge. This was done in order to make the leak back through the ionized gas as small as possible. The time from the closing of $T_{2}$ to the opening of $K_{2}$ was about .I sec. The charge on $C$ was now measured by closing the key $K_{1}$ and noting the deflection on the electrometer. The operation was now repeated with the potential across $B-A$ for .2 sec., giving a second charge consisting of the $N$ as before plus the charge produced for .2 sec. Thus by taking several successive equal intervals of time, throws were obtained which when carried back to zero time gave the throw corresponding to $N$. A complete set of such readings for air at $100 \mathrm{~mm}$. pressure is given in Table I and the resulting curve by the dotted line in Fig. 2. The throws as indicated on the electrometer need two corrections; one for the loss of charge due to the leak back through the ionized gas during the . I sec. elapsing between the closing of $T_{2}$ and the opening of $K_{2}$; the second for the leak from the plate $A$ to the guard ring, which begins to come in 
when $T_{1}$ is opened and continues with increasing value until $K_{2}$ is opened. The amount of these corrections depends upon the potential to which $C$ and $A$ are charged and are therefore larger for the longer intervals. The full line in Fig. 2 shows the curve corrected for these two errors. It is seen there that although the corrections may be quite large they effect the value obtained for $N$ comparatively little, merely increasing the slope of the line. For the given pressure, $100 \mathrm{~mm}$. these errors were nearly at a maximum, being

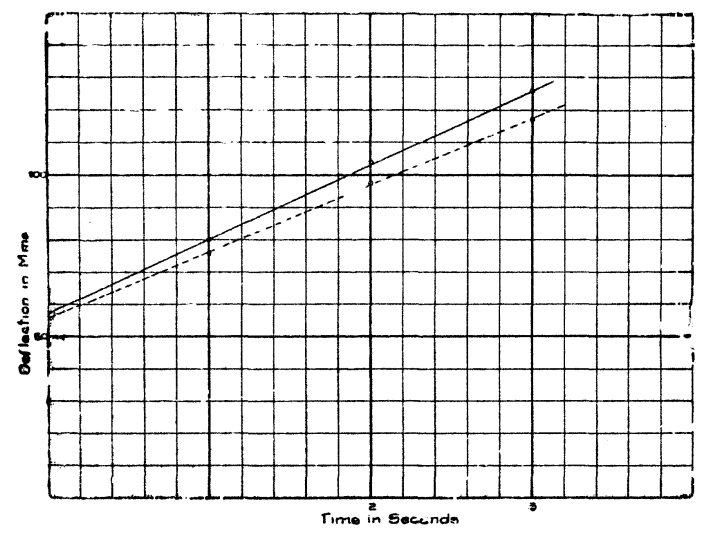

Fig. 2.

much smaller at higher pressures and not so very much larger at the smallest pressures used.

By this method of obtaining $N$, all disturbances, such as leaks across the electrodes, irregularities in the ionization due to secondary actions of the radiation at the plates or other causes, were eliminated, as they tended merely to effect the slope of the line and not the place where it cuts the axis. The regularity of the readings is shown by the example in Table I. These readings could be repeated at any time fairly closely.

\section{TABLE I.}

Pressure $100 \mathrm{~mm}$. Distance between plates $6 \mathrm{~mm}$. Volume 3435 c.c. Sensitiveness of electrometer 224 divisions for I volt on quadrants and $\mathbf{5 2}$ volts on needle.

For the saturation current $Q:$ Capacity $=.9$ microfarads. Volts across plates 440 . Time for 30 div., 14 secs. Leak to guard ring 1.6 divs. in 14 secs. 
$Q=31.6$ divs. in 14 secs. $=27.2$ E. S. units per sec.

For $N$ : Sensitiveness, 224 divs. for I volt. Capacity .0102 M.F.

\begin{tabular}{c|c|c|c}
\hline Time E.M.F. Acted Across Plates (Secs.). & \multicolumn{1}{|c|}{$\mathbf{1}$} & $\mathbf{. 2}$ & $\mathbf{. 3}$ \\
\hline & 76 & 97 & 117 \\
& & 97 & 118 \\
Scale divisions. & 76 & 96.5 & 119 \\
& 78 & 99 & 116.4 \\
& 76 & 97 & 116 \\
\hline Average. & 76.6 & 97.5 & 117.3 \\
Leak back through ionized gas. & 2.5 & 3.2 & 3.9 \\
Leak to guard ring. & .8 & 2.4 & 4.4 \\
\hline Corrected average. & 79.9 & 103.1 & 125.6 \\
\hline
\end{tabular}

Rate of leak back through ionized gas 32 divs./sec. at roo divs. deflection. Time of leak. I sec. Rate of leak to guard-ring 12 divs./sec. at Ioo divs. deflection.

$$
\begin{aligned}
\therefore N(\text { see Fig. } 2) & =57.2 \text { divs. }=7.76 \text { E. S. units. } \\
a & =\frac{\ell}{N^{2}} V=\mathbf{i}, 550 .
\end{aligned}
$$

Care was taken to have the air dry and dust-free by passing it through phosphorus pentoxide and cotton wool before letting it into the pump and vessel. Also the question of a saturation potential for $N$ had to be carefully looked into, as it was found that it took a higher potential to insure a maximum throw for $N$ than it did for $Q$ at the same pressure.

\section{Distribution of the Ionization.}

It is evident that with a certain rate of production the number of ions in the gas between the plates for a steady state will depend upon the distribution of the ionization. For by equation (I), the number of ions disappearing per sec. in one c.c. varies as the square of the number present, therefore a steady state will be reached with a smaller value of $N$ for large ionization gradients than for small ones. To take this into account it is necessary to know the law of the distribution of the ions.

Rutherford ${ }^{1}$ has shown experimentally that the intensity of the ionization due to a large plane surface of active matter, such as radium or uranium, falls off approximately according to an expo-

${ }^{1}$ Phil. Mag., Jan., I899; “'Radioactivity," p. 56. 
nential law with the distance from the plates, i.e., calling $q$ the rate of production of the ions per c.c. at a point distant $y$ from the active plate

$$
q=q_{0} e^{-\lambda y},
$$

where $q_{0}$ is the rate of production at the surface and $i$ the absorption constant. This result is deduced theoretically on the assumption that the ionization is proportional to the intensity of the radiation and that the energy of the rays is used up in producing ions. Bragg ${ }^{1}$ later on showed that this exponential law is not necessarily a true one, but might hold very closely for the complex radiations from radium spread over a large surface.

In the present case we have rather complex conditions, the ionization being due chiefly to the $\alpha$ rays from the radium on the two plates, but the $\beta$ rays and the radiation from the emanation throughout the volume of the gas also play some part. To obtain the actual distribution the exponential law was assumed and experiments made to find out how closely it held and for what value of $\lambda$.

Taking the origin of coördinates half way between the plates which are $2 a \mathrm{~cm}$. apart, and the axis $x$ at right angles to the plane of the plates, we have by the exponential law

$$
q=q_{0} c^{-\lambda(a-x)}+q_{0} e^{-\lambda(a+x)}
$$

each factor being the contribution of one plate to the ionization $q$ at the point $x$.

Taking the volume integral of $q$

$$
Q=\int q d v=A \int_{-a}^{+a} q_{0}\left[e^{-\lambda(a-x)}+e^{-\lambda(a+x)}\right] d x
$$

$A$ being the area of one plate.

Integrating,

$$
Q=2 A q_{0} \frac{\mathrm{I}-e^{-2 \lambda a}}{\lambda^{\prime \prime}}
$$

Using this equation and obtaining saturation currents for different distances between the plates it was found that the conditions were closely satisfied for the value $\lambda=.05, a$ being expressed in

\footnotetext{
' Phil. Mag., Dec., I 904.
} 
millimeters. This value of $i$ was obtained by taking any two distances with the corresponding $Q$ 's, giving two equations of the form of (6) from which $q_{0}$ was eliminated. Knowing $\lambda$ and starting with the $Q$ for $60 \mathrm{~mm}$. distance the other $Q$ 's can be calculated. These values are shown in Table II.

TABLE II.

Pressure $760 \mathrm{~mm}$.

\begin{tabular}{c|c|c}
\hline Distance Between Plates. & $\begin{array}{c}Q \\
\text { Observed. }\end{array}$ & $\begin{array}{c}Q \\
\text { Calculated for } \lambda=.05 .\end{array}$ \\
\cline { 2 - 3 } $60 \mathrm{~mm}$. & 106 & 106 \\
30 & 87 & 86.6 \\
20 & 70.8 & 70.8 \\
10 & 45.6 & 44 \\
\hline
\end{tabular}

The constant $\lambda$ has been found by Rutherford ${ }^{1}$ to vary approximately inversely as the density of the absorbing matter. Therefore calling $\lambda_{1}$ the constant at a pressure $p_{1}$ and $\lambda$ its value at 760 mm., we can write eq. (6), since $q_{0}$ also varies as the pressure

$$
Q_{1}=2 A q_{0}^{\prime}\left(\mathrm{I}-\epsilon^{-2 a \lambda p_{1} / 760}\right)
$$

Here $Q_{1}$ is the saturation current at the pressure $p_{1}$ and $q_{0}^{\prime}$ the rate of production per c.c. at the radiating surface at $760 \mathrm{~mm}$. pressure. This equation expresses the variation of the saturation current with the pressure of the gas. Observed values of this variation, also values calculated for $\lambda=.05$, are given in Table III.

TABLE III.

Plates $60 \mathrm{~mm}$. apart. $a=30 \mathrm{~mm}$.

\begin{tabular}{c|c|c}
\hline Pressure. & $\begin{array}{c}Q \\
\text { Observed. }\end{array}$ & Calculated for $\lambda=.05$. \\
\cline { 2 - 3 } & 106 & 106 \\
600 & 101 & 101.5 \\
450 & 94 & 94 \\
300 & 77 & 78 \\
200 & 55 & 60.7 \\
100 & 28.2 & 36.7 \\
50 & 14.2 & \\
\hline
\end{tabular}

" Radioactivity," p. 137. 
[Yor. XXI.

Fig. 3 shows these values plotted. The full line curve shows the observed variation of $Q$ with the pressure, the dotted line the calculated variation. At high pressure the coincidence is very close. Thus we are justified in assuming that at high pressures at least the distribution is given by the exponential law with .05 as the value of the absorption constant. Below $300 \mathrm{~mm}$. pressure the curve is nearly a straight line and the ionization is practically uniform, and so we need no correction.

The value of $\lambda=.05$ obtained here is much smaller than the value

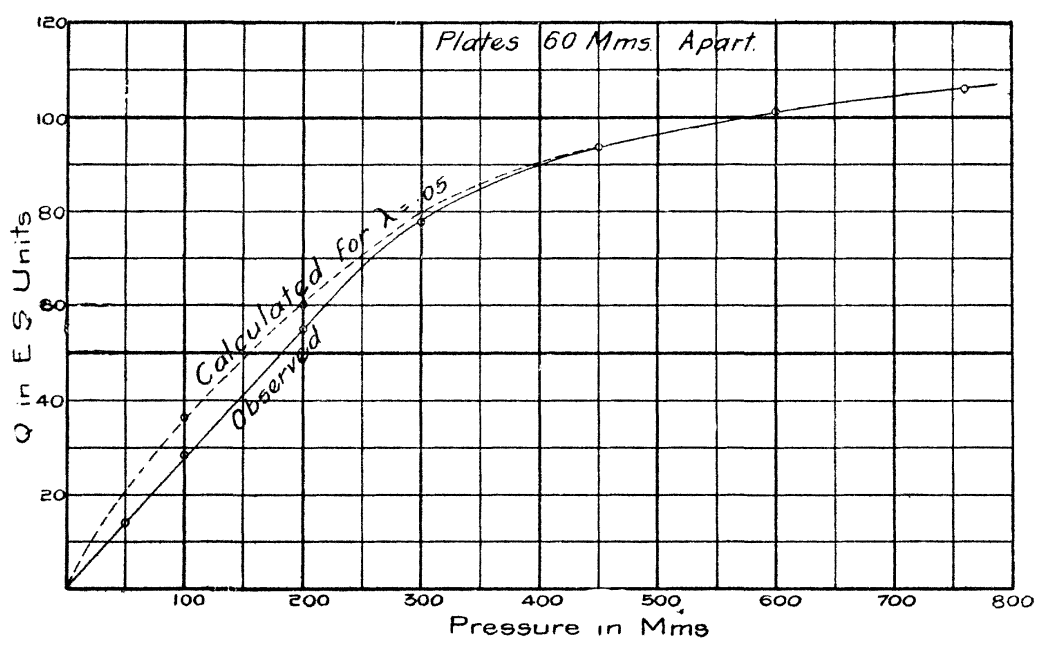

Fig. 3.

.9 given by Rutherford ${ }^{1}$ as the absorption constant for the $\%$-rays from radium. His value, however, was obtained by a somewhat different method, under different experimental conditions from the ones used here. In his work radiferous barium was used and the ionization measured after a good part of the radiation had been absorbed by passing through air and aluminium, thus getting a portion of the more easily absorbed $\alpha$-rays. Also the curves given by Rutherford ${ }^{2}$ for variation of the saturation current with the pressure between two large parallel plates, one of which was active, fall

\footnotetext{
1 " Radioactivity," p. I 37 .

${ }_{2}$ Phil. Mag., Jan., I899, pp. I $30,{ }_{3} 8$.
} 
off much more rapidly than his theory would indicate, using such a large value of $\lambda$.

\section{Correction to $\alpha$ For Uneven Distribution of the Ionization. ${ }^{1}$}

Suppose $n$ as before to be the electric density of the free charge at the point $x$ when a steady state has been reached. Taking the volume integral

$$
N=\int n d v
$$

but by equations (3) and (5)

$$
\left.n=\sqrt{\frac{q}{\alpha}}=\sqrt{\frac{q_{0}}{\alpha}\left(e^{-\lambda(a-x)}\right.}+e^{-\lambda(a+x)}\right)
$$

therefore

$$
N=A\left(\frac{q_{0}}{\alpha}\right)^{\frac{1}{2}} \int_{-a}^{a}\left[e^{-\lambda(a-x)}+e^{-\lambda(a+x)}\right]^{\frac{1}{2}} d x .
$$

But as the ionization is symmetrical out from the middle point toward either plate the lower limit can be changed from $-a$ to 0 if the integral is multiplied by the factor 2. Doing this, and rewriting

$$
N=2 A\left(\frac{q_{0}}{\alpha}\right)^{\frac{1}{2}} e^{-\frac{1}{2} \lambda a} \int_{0}^{a} e^{\frac{1}{2} \lambda x}\left(\mathrm{I}+e^{-2 \lambda x}\right)^{\frac{1}{2}} d x .
$$

Expanding $\left(\mathrm{I}+e^{-2 \lambda x}\right)^{\frac{1}{2}}$ by the Binomial Theorem, multiplying by $e^{\frac{1}{2} \lambda x}$, integrating the series term by term and simplifying we have

$$
N=2 A\left(\frac{q_{0}}{a}\right)^{\frac{1}{2}} \frac{1}{\lambda}\left[\left(4-\frac{2}{3} e^{-2 \lambda a}+\frac{1}{14} e^{-4 \lambda a}++\right)-e^{-\frac{1}{2} \lambda a}\left(4-\frac{2}{3}+\frac{1}{14}++\right)\right] .
$$

Since in these experiments $\lambda$ is .05 and $a 30, \lambda a$ has the value. I 5 , and both of these series are rapidly converging, therefore a close approximation is obtained by neglecting all except the first two terms of the first series and taking 3.38 as the sum of the second. Thus

$$
N=2 A\left(\frac{q}{a}\right)^{\frac{1}{2}} \frac{4-\frac{2}{3} e^{-2 \lambda a}-3 \cdot 38 e^{-\frac{1}{2} \lambda \iota}}{\lambda} .
$$


But from equation (6)

$$
q_{0}=\frac{\lambda Q}{2 A\left(\mathrm{I}-e^{-2 \lambda a}\right)} .
$$

Substituting this value and solving for $\alpha$

$$
\alpha=\frac{Q}{N^{2}} A \frac{\left(4-\frac{2}{3} e^{-2 \lambda a}-3 \cdot 38 e^{-\frac{1}{2} \lambda a}\right)^{2}}{2 \lambda\left(\mathrm{I}-e^{-2 \lambda a}\right)} .
$$

The exponential term in this equation should be equal to 6 , the number of $\mathrm{cm}$. between the plates for uniform ionization. For the value $\lambda a=.15$ this term becomes equal to 5.9. Therefore even at atmospheric pressures the correction for uneven distribution is very small and $\alpha$ can be taken equal to $Q V / N^{2}$ where $V$ is the volume of gas between the plates.

\section{Effect of Diffusion at Low Pressures.}

At above $100 \mathrm{~mm}$. pressure it was found that with the plates $6 \mathrm{~cm}$. apart the loss of ions due to diffusion to the plates and sides of the vessel was negligibly small compared with the loss due to recombination proper. Below this pressure however the observed $N$ began to decrease very rapidly due to diffusion and

$$
\frac{Q}{N^{2}} V
$$

to increase correspondingly. It is easily seen how this would come about, since if the loss of ions due to diffusion is appreciable a steady state will be reached for a smaller value of $N$ than it would if there was no such loss.

Due to diffusion there will be an ionization gradient along $x$ from the plane midway between the plates out to their surfaces, where there will be no ionization, the ions there present giving up their charges to the plates. The charge on the ions crossing unit area in unit time due to the gradient $d n / d x$ will be $D d n / d x$, where $D$ is the coefficient of diffusion. Therefore the rate of increase of free charge in any unit volume will be $D d^{2} n / d x^{2}$. Thus for a steady state

$$
q=\alpha n^{2}-D \frac{d^{2} n}{d x^{2}}
$$


A satisfactory solution of this equation is not easily found, but for the special case where the factor due to recombination is negligibly small, J. J. Thomson ${ }^{1}$ gives the solution

$$
n=\frac{1}{2} \frac{q}{D}\left(a^{2}-x^{2}\right),
$$

from which

$$
N_{0}=\frac{1}{3} \frac{q a^{2}}{D}
$$

$2 a$ being the distance between the plates.

This $N_{0}$ represents the charge on the ions which would be in the gas for a steady state if the ions disappeared by diffusion only. The coefficient of diffusion $D$ has been shown by Townsend ${ }^{2}$ to vary inversely as the pressure down to $200 \mathrm{~mm}$. so that the loss due to diffusion increases as the pressure decreases. Also it is seen that the diffusion effect varies inversely as the square of the distance between the plates.

This rapid increase of the diffusion effect as the plates get close together was used to get an idea of the error in the observed $N$ brought in by diffusion.

A series of determinations of $N$ and $Q$ were made for different distances between the plates. These results are shown for pressures of 50,20 and $10 \mathrm{~mm}$. in tables IV.-VI. The fourth column of these tables show the very rapid rise, with the small distances, of the quantity $Q / N^{2} V$ which would be $\alpha$, the coefficient of recom-

TABLE IV.

Area of Plate, $572 \mathrm{sq} . \mathrm{cm}$. Pressure, $50 \mathrm{~mm}$.

\begin{tabular}{c|c|c|c|c}
\hline $\begin{array}{c}\text { Distance Between } \\
\text { Plates. }\end{array}$ & $Q$. & $N$. & $\frac{Q}{N^{2}} V=\alpha^{\prime}$. & $N_{D .}$ \\
\hline $8 \mathrm{~cm}$. & 16 & 7.4 & 1,340 & 160 \\
6 & 14.2 & 5.9 & 1,380 & 80 \\
3 & 7.64 & 2.9 & 1,570 & 16 \\
2 & 5.7 & 1.9 & 1,830 & 4 \\
\hline
\end{tabular}

Limiting value of $a^{\prime}$ is $a=1,310$.

${ }^{1}$ J. J. Thomson, "Conduction of Electricity through Gases," p. 20. 2 Townsend, Phil. Trans., A, r95, p. 2 9, 1900. 
TABLE V.

Pressure, $20 \mathrm{~mm}$.

\begin{tabular}{c|c|c|c|c}
\hline $\begin{array}{c}\text { Distance Between } \\
\text { Plates. }\end{array}$ & $Q$. & $N$ & $Q \begin{array}{c}Q \\
N^{2}\end{array}$ & $N_{D .}$ \\
\hline $8 \mathrm{~cm}$. & 6.4 & 4.75 & 1,290 & 25.6 \\
6 & 4.7 & 3.14 & 1,600 & 10.5 \\
3 & 3.0 & 1.14 & 4,000 & 1.7 \\
2 & 2.3 & .52 & 10,000 & .58 \\
\hline
\end{tabular}

Limiting value of $a^{\prime}=a=1,150$.

TABLE VI.

Pressure, $10 \mathrm{~mm}$.

\begin{tabular}{c|c|c|c|c}
\hline $\begin{array}{c}\text { Distance Between } \\
\text { Plates. }\end{array}$ & $Q$. & $N$. & $N^{2} V=a^{\prime}$. & $N_{D .}$ \\
\hline $8 \mathrm{~cm}$. & 3.1 & 2.7 & 2,100 & 6.2 \\
6 & 2.4 & 1.4 & 4,000 & 2.7 \\
3 & 1.6 & .38 & 19,000 & .45 \\
2 & 1.21 & .143 & 68,000 & .15 \\
\hline
\end{tabular}

Limiting value of $a^{\prime}=a=1,000$.

bination, if all the loss of ions was considered due to recombination. It is evident that if there was no diffusion, this quantity $Q / N^{2} V$, which will be called the apparent $\alpha$ and denoted by $\alpha^{\prime}$ would be independent of the distance between the plates.

To obtain $\alpha$ at each pressure a curve was plotted between $\alpha^{\prime}$ as ordinate and the distances between the plates as abscissa, and the value of $\alpha^{\prime}$ for which this curve became a straight line parallel to the horizontal axis was taken as the true coefficient of recombination. These curves are shown in Fig. 4.

At $50 \mathrm{~mm}$. pressure it is seen that diffusion makes very little error in $\alpha$ even when the plates are $2 \mathrm{~cm}$. distance apart, while at IO $\mathrm{mm}$. pressure for the small distances between plates it has a very great effect, the loss of ions due to diffusion evidently exceeding that due to recombination. The values of $\alpha$ finally adopted and given in Tables IV.-VI. were taken from these curves plotted on a much larger scale.

A good idea of the way in which diffusion and recombination enter at the different pressures and distances may be obtained by a 
consideration of the experimental values of $N$ in the Tables IV.VI. and the values given in the last column headed $N_{D}$. This $N_{D}$ is the value of $N$ which would have been expected if the loss of the ions balancing the rate of production $Q$ was due to diffusion only. They are calculated from equation (8) taking the coefficient of diffusion $D$ at Townsend's value of .035 for atmospheric pressure and assuming it to vary inversely with the pressure. The larger the

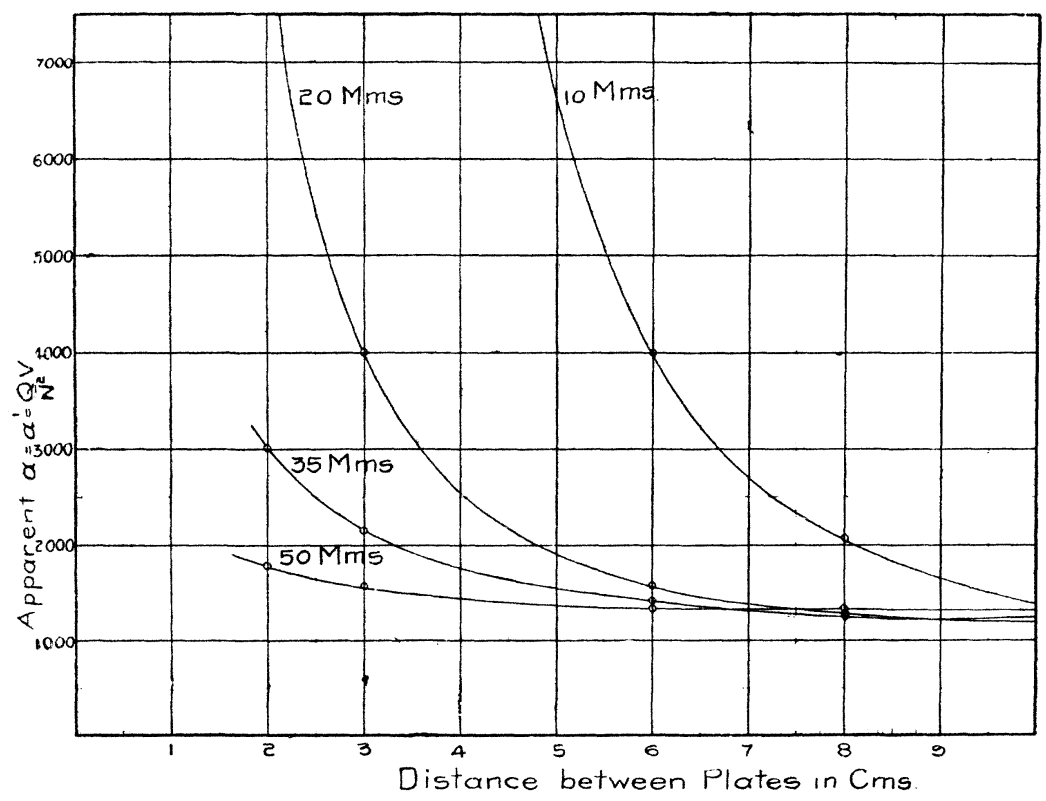

Fig. 4.

value of $N_{D}$ compared with $N$ the less the effect of diffusion. Thus at $50 \mathrm{~mm}$. pressure and the plates $8 \mathrm{~cm}$. apart, diffusion plays very little part, but at $10 \mathrm{~mm}$. pressure for the plates $2 \mathrm{~cm}$. apart it accounts for nearly the entire loss of ions.

For pressures below $10 \mathrm{~mm}$. pressure and with vessels of ordinary dimensions as in discharge tubes, for instance, practically all of the ions disappear by diffusion to the sides of the vessel.

The Coefficient of Diffusion at Low Pressures.

For $20 \mathrm{~mm}$. and $\mathrm{IO} \mathrm{mm}$. pressures and for the small distances between the plates diffusion has been seen to play the important 
part. Proceeding in a manner analogous to the method adopted for getting $\alpha$ separate from the diffusion error, it is possible to get a fairly good value for the coefficient of diffusion $D$ at these pressures. Thus it is evident that the coefficient of diffusion, which is given by the equation

$$
D=\frac{1}{3} \frac{Q}{N} a^{2},
$$

when there is no recombination, is a constant at any pressure and should not depend upon the distance between the plates. If, using the experimental values for $Q$ and $N, D$ apparently increases with the distances between the plates, it means that, due to recombination, the experimental $N$ is smaller than it would be for diffusion loss only. The relative loss due to recombination should increase with the distances between the plates. Fig. 5, plotted from the values in the tables VII. and VIII. shows the increase of this apparent $\bar{D}$ with the distances between the plates.

TABLE VII.

Pressure, $20 \mathrm{~mm}$.

\begin{tabular}{c|c|c|c}
\hline Distance Between Plates. & $Q$. & $N$. & $\frac{1}{3} \frac{Q}{N} a^{2}=D^{\prime}$. \\
\hline $8 \mathrm{~cm}$ & 6.4 & 4.75 & 7.1 \\
6 & 4.7 & 3.14 & 4.4 \\
3 & 3.0 & 1.14 & 1.9 \\
2 & 2.3 & .52 & 1.4 \\
\hline
\end{tabular}

TABLE VIII.

Pressure, $10 \mathrm{~mm}$.

\begin{tabular}{c|c|c|c}
\hline Distance Between Plates. & $\varrho$. & $N$. & ${ }^{\frac{1}{3}} Q_{N} a^{2}=D^{\prime}$. \\
\hline $8 \mathrm{~cm}$ & 3.4 & 2.7 & 6.8 \\
6 & 2.38 & 1.4 & 5.0 \\
3 & 1.6 & .38 & 3.2 \\
2 & 1.21 & .143 & 2.8 \\
\hline
\end{tabular}

As in the curves for $\alpha$, where the $D^{\prime}$ becomes constant with the distance between the plates is taken as the coefficient of absorption at that pressure. In this manner $D$ was found to have the value I I. at $20 \mathrm{~mm}$. pressure and 2.6 at $10 \mathrm{~mm}$. 
Reducing these values back to atmospheric pressure on the assumption that $D$ varies inversely with the pressure, we get the values .030 and .034. Townsend found (loc. cit.) that $D$ varied inversely with the pressure down to $200 \mathrm{~mm}$. pressure and his value at atmospheric pressure was .035. The two values given above

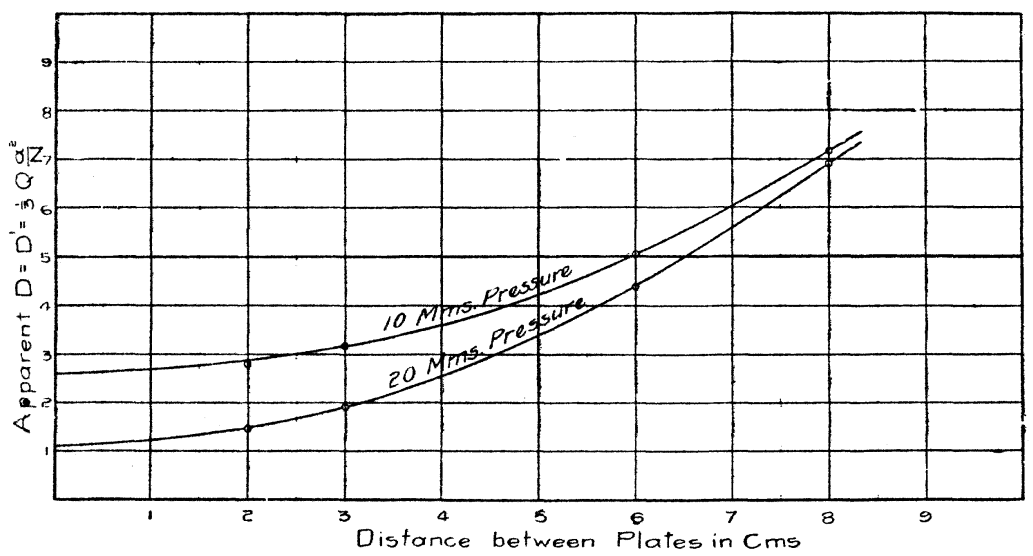

Fig. 5.

thus indicate that $D$ continues to vary inversely with the pressure at least down to $10 \mathrm{~mm}$.

As, by theory, $D$ varies directly with the specific velocities of the ions this would indicate that the velocities too are a linear function of the pressure down to $10 \mathrm{~mm}$. J. Stark ${ }^{1}$ gives some results showing this, but Langevin ${ }^{2}$ has found that the velocities increase with decrease of pressure faster than a linear law would allow.

\section{Variation of $\alpha$ with the Pressure.}

The complete results for the variation of $\alpha$ with the pressure are given in Table IX. and plotted in the form of a curve in Fig. 6. The values of $\alpha$ here given are the mean of several determinations. The values of $N$ given in the table are not in every case the ones from which the corresponding $\alpha$ was obtained, as some of the determinations were made before the radium had reached a steady state.

1 J. Stark, Die Electricität in Gasen, p. 26r.

$2 \mathrm{M}$. Langevin, Thèse, p. I 40 . 
TABLE IX.

$V=3,435$ c.c.

\begin{tabular}{c|c|c|c}
\hline Pressure. & $\varrho$ & $N$ & $a=\frac{Q}{N^{2}} V$. \\
\hline $760 \mathrm{~mm}$ & 106 & 10.5 & 3,300 \\
450 & 94 & 11 & 2,650 \\
250 & 67 & 10.6 & 2,070 \\
150 & 42.2 & 9.1 & 1,750 \\
100 & 28 & 7.9 & 1,550 \\
50 & 14.2 & 6.1 & 1,310 \\
35 & 10 & 5.2 & 1,250 \\
20 & 5.6 & 4.1 & 1,150 \\
10 & 3.0 & 3.2 & 1,000 \\
\hline
\end{tabular}

At atmospheric pressure the value of $\alpha$ has been previously well fixed. Townsend obtained 3,400, McClung 3,384 and Langevin 3,200 as the absolute value of $\%$. The value 3,300 given here is seen to agree very closely with these results.

As to the variation of $\%$ with the pressure the curve in Fig. 6

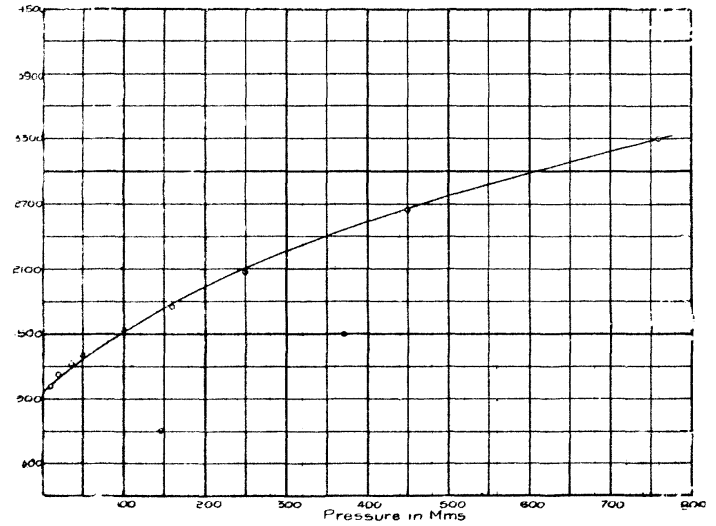

Fig. 6.

Cuefficient of Recombination $a$.

shows that $\alpha$ decreases to a value I,O0O at Io $\mathrm{mm}$. pressure, the rate of decrease constantly increasing as the pressure falls.

The curve falls below that of McClung's who found $\alpha$ independent of the pressure down to about $100 \mathrm{~mm}$. It goes above that of Langevin who found a somewhat more rapid decrease. Lan- 
gevin does not give the absolute value of $\alpha$ obtained at pressures below atmospheric, but does give the data for 375 and $152 \mathrm{~mm}$. for which the values 1,500 and 600 have been calculated. These values are indicated in Fig. 6 by two $\times$ marks.

It is difficult to compare the present results as to accuracy with those of Langevin as his experimental work was based on a rather complicated theory. In a general way Langevin's results are confirmed as there is a decrease of $\alpha$ with the pressure.

With McClung's results however a comparison can be made as both methods were direct ones. Langevin makes the following legitimate criticisms of the experimental conditions under which McClung worked.

The intensity of the Röntgen rays vary considerably in very short intervals of time, thus making it necessary to take the average of very many widely divergent readings. Also the ionization is very small even at high pressures, rendering accurate determinations of the charges difficult.

An error is introduced by not knowing the exact distribution of the ionization. Also the effect produced by the secondary radiations emitted by the metal plates under the action of the Röntgen rays is considerable and continues after the Crookes tube has been stopped.

Loss of ions by diffusion to the electrodes also brings in a probable error.

This last source of error might explain to some extent McClung's large values of $\alpha$ for low pressures, as he had a very large surface exposed and the electrodes were close together. This diffusion effect has been shown to come in very rapidly as the distance between the plates and the pressure decrease.

Practically all of these objections except that due to uneven distribution have been met in the present work. The intensity of the radium radiations was very constant and the ionization large and thus the readings could be repeated within a few per cent. The secondary radiations could introduce no error on account of the method of determining $N$. The diffusion effect was corrected for in those cases where it was appreciable. As for the distribution error, it has been shown that with a somewhat large divergence 
from a uniform distribution the correction for $\alpha$ is very small, therefore any error in the determination of the distribution could not effect the value of $\alpha$ very much.

In conclusion I wish to acknowledge my indebtedness to Dr. Bergen Davis at whose suggestion this investigation was taken up and with whose advise it was carried on.

Phonix Physical Laboratory,

Columbia University, May, 1905. 